\title{
Fugitive hydrocarbon emissions from Indiana oil/gas wells into the atmosphere
}

YIN, Yidong ${ }^{1}$, SCHIMMELMANN, Arndt ${ }^{1}$ MASTALERZ, Maria ${ }^{2}$ and DROBNIAK, Agnieszka ${ }^{2}$

(1) Department of Earth and Atmospheric Sciences, Indiana University, $1001 \mathrm{E}$. 10th St., Bloomington, IN 47405; (2) Indiana Geological and Water Survey, 611 N. Walnut Grove Ave., Bloomington, IN 47405

reductio

Hydrocarbon gas emissions from oil and gas wells contribute significantly to

Methe flux from oil and gas wells has

Methe

methane emissions from oil/gas wells to mitigate global warming

There are more than 80,000 active, inactive, temporarily abandoned and permanently

plugged oil and gas wells in Indiana. The extent of fugitive hydrocarbon emissions from

those wells into the atmosphere has not been quantitatively studied. This study is a first attempt to quantify those emissions.

Methanotrophs are methane-oxidizing bacteria which can metabolize methane. Methane in natural seepages can be partly consumed by methanotrophs in soil around

abages before it reaches the atmosphere [5]. Therefore, solis enriched in methanotrophs

above hydrocarbon leakages have a potentlal to milgale hydrocarbon emissions from

rtificial soil mound experiments.

Experiments

. Research area

Wed 20 active, temporarily deactivated or Wullivancounties in Indian. Fugitive anissions on

.

Monroe County to serve as our research site.

Quantification of methane emission

We built a polyethylene tent to enclose fittings of a

leaking research well. The bottom of the polyethylene

foil was ballasted by stones to seal against the ground. $A$

DC-operated fan homogenized the methane

cencentration in the tent. Air from within the tent was

sampled by a SARAD RTM 2200 instrument and metha

concentrations were determined with an Axetris laser

OEM Module $\mathrm{LCF} 20 \mathrm{CH}_{4}$ detector. The methane

(

$\left(\mathrm{ml} \mathrm{m}^{-3} \mathrm{~m}^{-1}\right)$ and $\mathrm{V}$ is volume of the tent $\left(\mathrm{m}^{3}\right)$

3. Mesocosm experiment

We collected topmost soils adjacent to and $20 \mathrm{~m}$ away

(control soils) from the research well and set up triplici

mesocosm systems of those soils in stoppered 70-mL

lass culture bottles in contact with a methane-enhanced

standard atmosphere. We measured the remaining

methane concentration over time in headspace of culture

bottles. We also measured changes in methanotrophic

activity of soil as a function of moisture content. The

original soil's moisture content was $32 \mathrm{wt} . \%$.

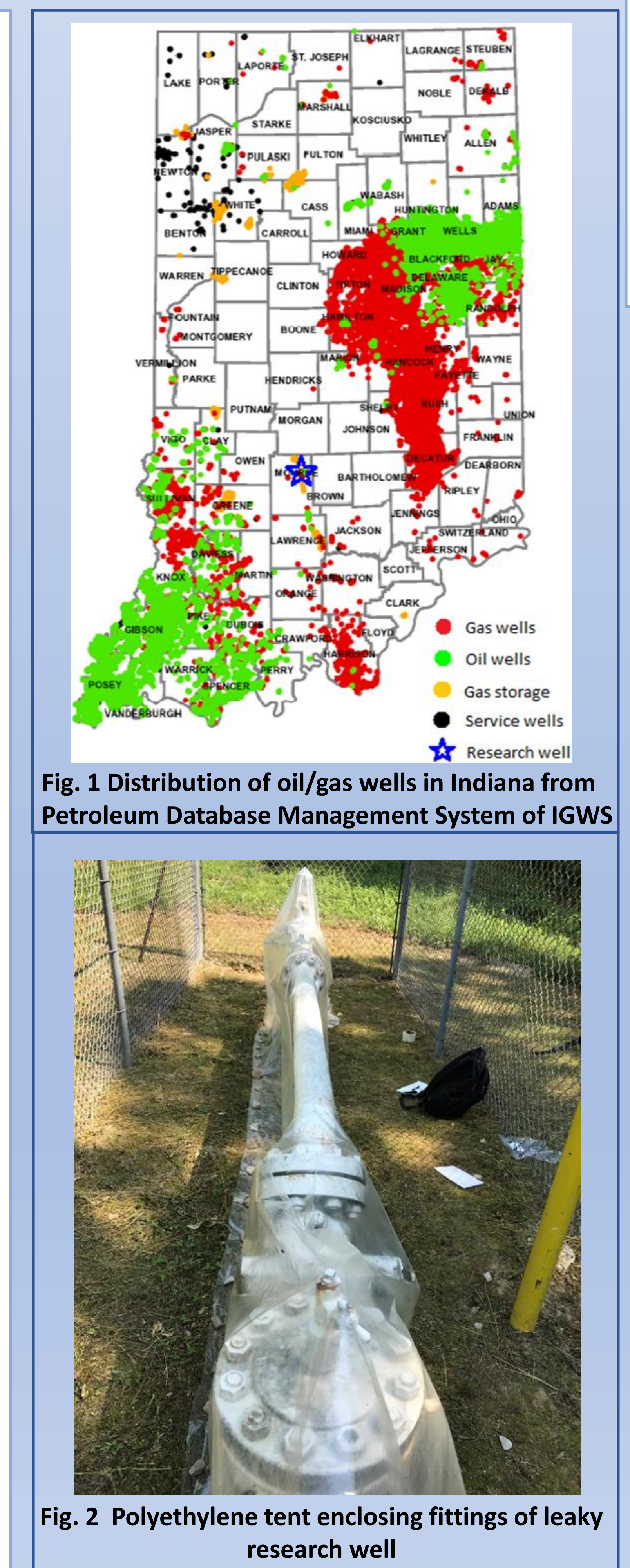

Experiments (continued)

4. Soil mound experiment

We

Whe

used soils from a solid waste landfill in Monroe

County to build a "methanotrophic mound" in

bucket and placed it in a $0.903 \mathrm{~m} \times 0.462 \mathrm{~m} \times 0.358$

$\mathrm{m}$ terrarium. The mound's base was a permeable

layer of rocks covered by soil. The height of the

mound was $0.15 \mathrm{~m}$ with a diameter of $0.258 \mathrm{~m}$. A

capillary tube was fixed at the bottom of the rock

layer and connected to a $\mathrm{CH}_{4}$ gas cylinder to

was $\mathrm{res}$.

was regulated by a control valve, which was set at $74.34 \mathrm{~mL} / \mathrm{h}$ (6 bubbles/min). The terarim was

sealed using an adhesive tape and the $\mathrm{CH}_{4}$

2200. We also tested if prolonged exposure of

mound (using cont soil from the leaking well) to

$\mathrm{CH}_{4}$ supplied in abundance will increase its

methanotrophic activity.

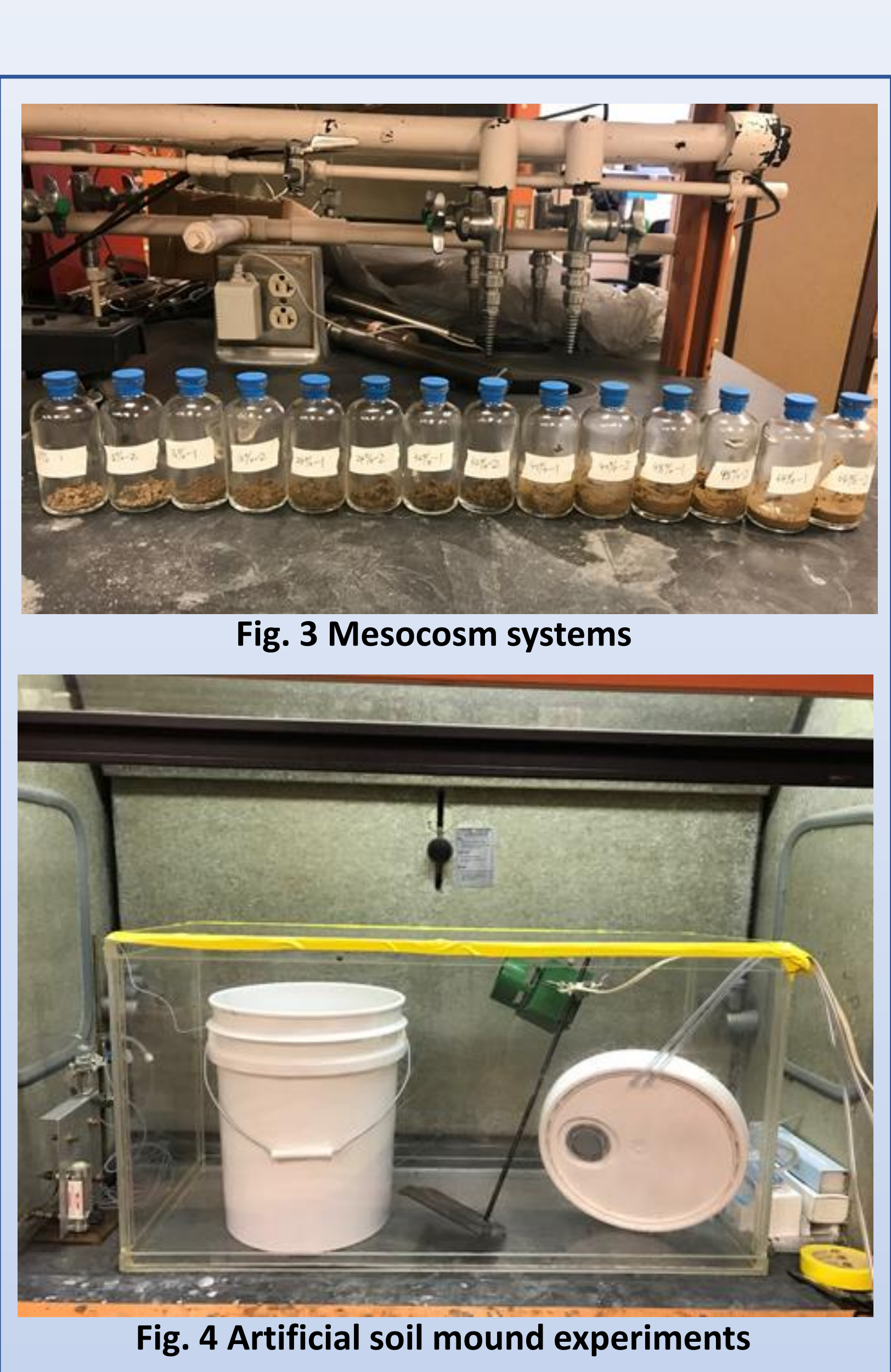
Results (continued)

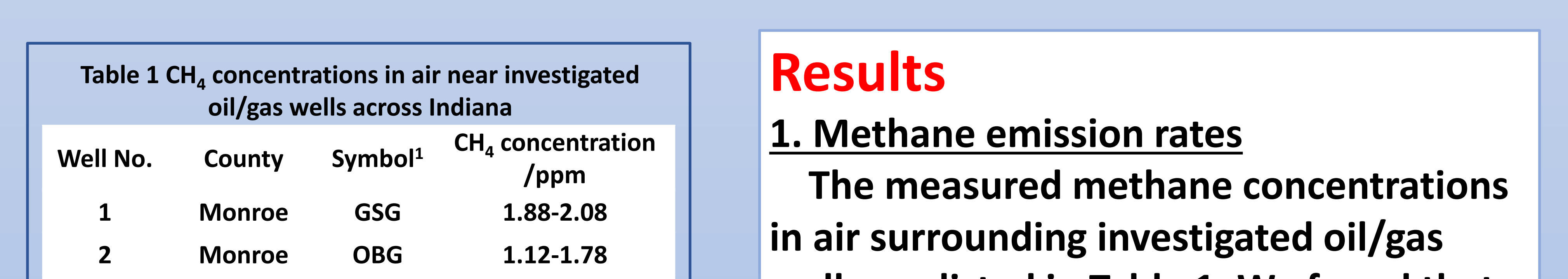

wells are listed in Table 1. We found that

methane concentrations in the tent
around the research well increased

linearly with time. The average methan

emission rate was calculated as

$1.73 \mathrm{~L} /$ hour using $\mathrm{R}=\mathrm{k} * \mathrm{~V} * 3.6$ ( $\mathrm{k}$ is 1.622

$1.323,1.366 \mathrm{~mL}^{*} \mathrm{~m}^{-3 *} \mathrm{~s}^{-1}$, respectively

the three measurements; $\mathrm{V}=0.335 \mathrm{~m}^{3}$ ).

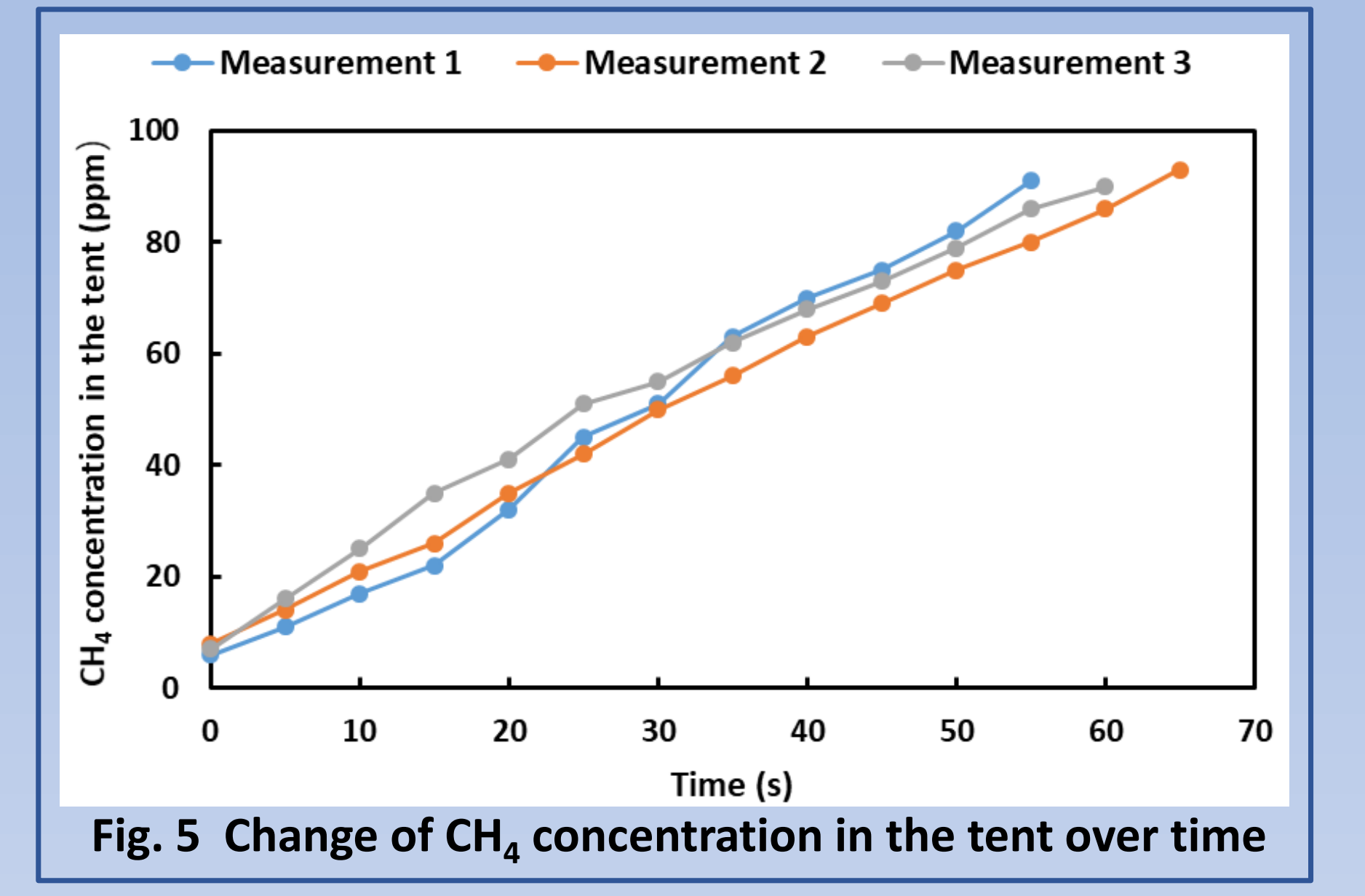

2. Mesocosm system

from the leaky well consumed 96

$\%$ of the initial $\mathrm{CH}_{4}$ after 138 hours, while the

After control soil was continuously exposed

to elevated methane concentrations for on

month in a culture bottle, its microbes

expressed enhanced methanotrophy relative to

those in the original control soil.

The order of soil moisture contents from

higher to lower methanotrophic activity is 16

$>22>24>40>48=64>0 \mathrm{wt}$. \%, indicating the

optimal moisture content with maximum

totally dried soil from adjacent to the leaky

well "woke up" the fastest, also in the soil

remoistened by $16 \mathrm{wt}$ \% added water.

Coil expresses dormant and

in wet soil does not allow easy access of

methane and oxygen from air to enter the sol

in

impeded.

3. Soil mound

methanotrophic activity can mitigate methan

emission more effectively compared to co

- The methanotrophic activity of the contro

soil mound can be enhanced over time after

being exposed to abundant methane (85 ppm) in the terrarium.
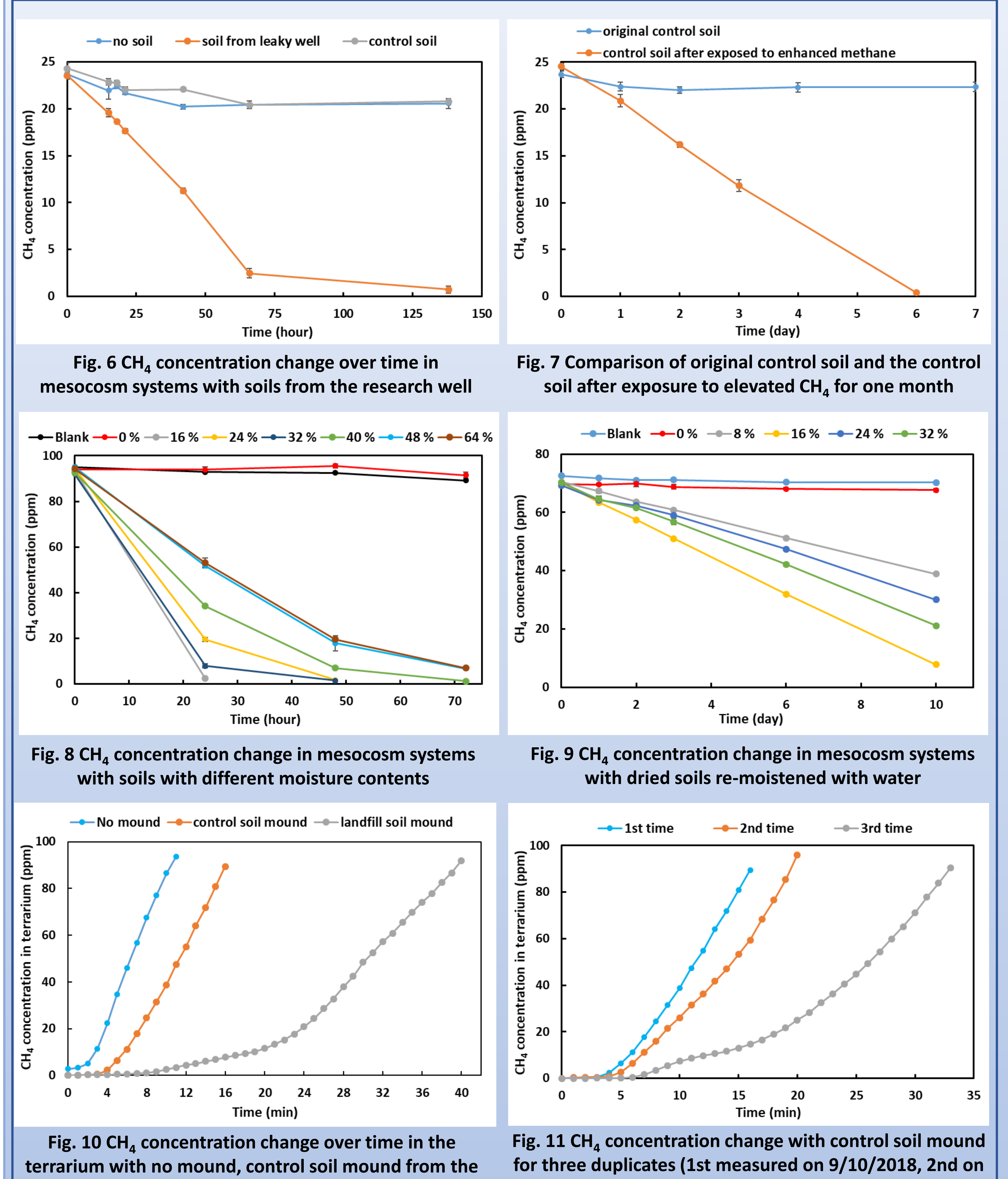

\section{References}

Al., 2016. Geophys. Res. Lett, 43: 2283-2290,

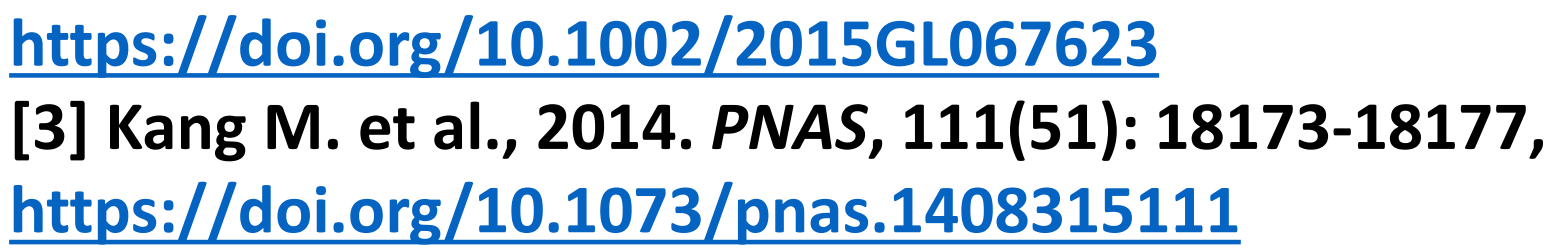

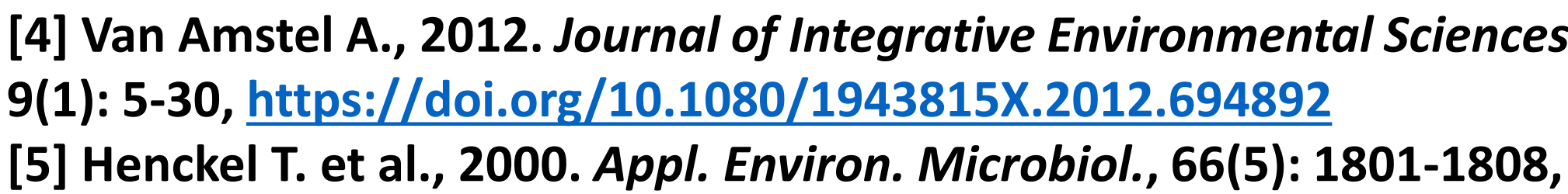

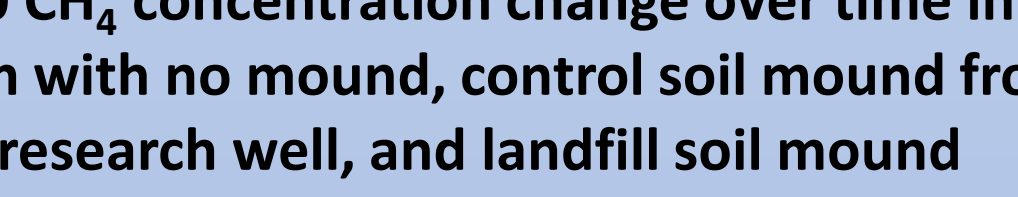

Conclusions

- The methane flux from the leaky research well is ca. 2

L/hour.

- Soils adjacent to leaking wells exhibit significant

methanotrophic activity. Prolonged exposure of soil

methanotrophs to elevated methane con

enhances their methanotrophic activity.

above abandoned and leaking oil/gas wells can be a cost-

effective strategy to microbially mitigate fugitive

hydrocarbon emissions from wells. 